\title{
EXTRACTIVISMO CLÁSICO Y NEOEXTRACTIVISMO, ¿DOS TIPOS DE EXTRACTIVISMOS DIFERENTES? \\ I Parte
}

\author{
Por: Luis Hernando Portillo Riascos ${ }^{1}$
}

\section{RESUMEN}

El presente trabajo tiene como objetivo fundamental aportar al debate sobre las diferencias entre dos tipos de extractivismo: clásico o convencional y, neoextractivismo. Con este propósito, se presenta, en una primera parte, los distintos aportes existentes en la literatura alrededor de este tema, y en una segunda parte, se hace un análisis de los principales cambios introducidos en el marco regulatorio del sector petrolero de los dos países estudiados, que son: Ecuador y Colombia.

Por los aportes derivados de la revisión de literatura y el análisis de los modelos de explotación petrolera, la diferencia fundamental viene dada por el rol que asume el Estado. Los cambios introducidos en este sentido fueron observados para el caso ecuatoriano. Los rasgos más importantes son: en primer lugar, el Estado busca asumir un mayor control de la actividad petrolera, y en segundo lugar, fruto de lo anterior, hay un cambio trascendental de las relaciones con las empresas transnacionales. Los elementos mencionados guardan correspondencia con los aportes citados bajo el neoextractivismo. Empero, aunque las modificaciones incorporadas en el marco regulatorio denotan grandes diferencias en los modelos de explotación petrolera de los dos países, es necesario evaluar la dinámica productiva y fiscal con el objeto de conocer el verdadero impacto de las medidas adoptadas en los dos países.

Palabras clave: extractivismo, neoextractivismo, modelos de explotación petrolera, Colombia, Ecuador.

Clasificación JEL: O13, Q33, Q48.

1. Economista y Especialista en Finanzas de la Universidad de Nariño. Máster en Economía Internacional y Desarrollo de la Universidad Complutense de Madrid. Docente tiempo completo adscrito al Programa de Economía de la Universidad de Nariño. E-mail: her182000@yahoo.es. Agradezco los comentarios y observaciones de María José Paz Antolín. 


\title{
CLASSIC AND NEOEXTRACTIVISMO EXTRACTIVISM, TWO DIFFERENT TYPES OF EXTRACTIVISMOS?
}

\author{
(Part I)
}

\author{
By: Luis Hernando Portillo Riascos
}

\begin{abstract}
This work has as its main objective, to contribute to the debate on the differences between two types of extractivism: classic or conventional and neoextractivismo. Whit this purpose, it presents, in a first part, the different existing contributions to itself in the literature about this topic, and in a second part, made an analysis of the main changes introduced in the regulatory framework of the oil sector in the two countries studied are: Ecuador and Colombia.

By the contributions derived from the literature review and analysis models of oil exploitation, the fundamental difference is given by the role assumed by the State. The introduced changes in this sense were observed for the Ecuatorian case. The most important features are: first, the State seeks to assume greater control of the oil industry, and secondly, as result of the above, there is a major change in relations with transnational corporations. The mentioned elements keep interrelation with the contributions quoted under the neoextractivismo. However, although the modifications incorporated into the regulatory framework denote significant differences in the models of oil exploitation of the two countries, is necessary to evaluate the dynamics and fiscal productive in order to know the true impact of the measures adopted in the two countries.
\end{abstract}

Key words: extractivism, neoextractivismo, oil exploration models, Colombia, Ecuador.

JEL classification: $\mathrm{O} 13, \mathrm{Q} 33, \mathrm{Q} 48$ 


\section{INTRODUCCIÓN}

El papel que han jugado los recursos naturales (RRNN) en la estructura y dinámica económica mundial es fundamental. Muchos de los países que hoy se consideran como desarrollados aprovecharon su dotación de RRNN para impulsar las primeras fases de su proceso de crecimiento económico. Sin embargo, los resultados alcanzados en distintos lugares no son los mismos; mientras algunos han sabido aprovechar dicha dotación, convirtiéndola en una palanca del crecimiento, en otros, su posesión más bien se ha transformado en su propio lastre.

América Latina (AL), históricamente, ha estado ligada a la explotación de RRNN. Se inserta a la división internacional del trabajo como proveedora de materias primas, bajo un carácter dependiente, y por tanto, sujeta a las necesidades de quienes demandan sus productos. La relevancia de este aspecto se puede vislumbrar en los flujos de Inversión Extranjera Directa (IED) y el tipo de exportaciones. En el primer caso, lo que se observa es que, desde 1970 en adelante, la IED mundial con destino a materias primas de origen primario se concentra en países subdesarrollados, los cuales tienen una participación promedio, entre 1970 y 2011, del 75\% del total ${ }^{2}$. En cuanto a las exportaciones de AL, aquellas de origen primario, representaban, en 1970, el 89.2\%, mientras que para 2011, la participación había bajado al 60.7\%; no obstante, continua siendo elevada ${ }^{3}$.

La riqueza de RRNN y las características de su patrón histórico de inserción externa, reproducen este modelo de explotación. Las reservas existentes y la importancia estratégica que adquieren en el marco de la globalización, lo profundizan aún más.

Los países de Sudamérica poseen una de las mayores reservas minerales del planeta: un 65\% de las reservas mundiales de litio, un $42 \%$ de plata, un 38\% de cobre, un $33 \%$ de estaño, un $21 \%$ de hierro, un 18\% de bauxita y un 14\% de níquel. Se estima que el potencial minero es aún mayor ya que la información geológica disponible es parcial. También son importantes sus reservas petroleras, sobre todo tras la certificación de los crudos extra pesados de la Faja del Orinoco en la República Bolivariana de Venezuela. La región posee además alrededor de un 30\% del total de los recursos hídricos renovables del mundo, lo que corresponde a más del 70\% del agua del continente americano. (Altomonte et al., 2013: 7).

La gran pregunta que siempre ha salido a colación es por qué una región tan rica en RRNN tiene tantas dificultades para sentar un proceso de crecimiento económico de largo plazo. Dicha inquietud se hace más latente en los últimos años, dado que los precios de las materias primas han alcanzado niveles relativos muy elevados. Esta situación lleva a plantearse una interrogante que es muy válida, y tiene que ver con la preocupación que existe sobre si lo que se vive actualmente es una repetición del extractivismo clásico, o por el contrario, hay países donde

2. Si se toma como referencia el periodo 2001 - 2011, la media sería del $89 \%$ (Cálculos propios a partir de la base de datos de UNCTAD)

3. La participación se ve afectada por la importancia que adquieren países como Brasil y México. 
se están rompiendo aquellas barreras y limitantes que el mismo plantea. La aplicación de diferentes medidas de política económica en algunos países de AL abre el debate acerca del modelo más adecuado para la explotación de los RRNN, y particularmente del petróleo.

En el contexto del boom de las materias primas, que se inicia en la primera mitad de la década de los 2000, algunos países de AL han trazado políticas tendientes a profundizar las actividades de carácter extractivo. No obstante, se anotan diferencias importantes en los modelos desplegados en cada uno de los casos. A partir del contraste de dichos modelos de explotación de RRNN, Gudynas (2011a, 2011c, 2012) señala dos variantes o tipos de extractivismo: el clásico o convencional, y lo que denomina como "neoextractivismo". En el primer caso utiliza como ejemplos a países como Colombia y Perú, y en el segundo, Ecuador, Bolivia y Venezuela. A su juicio, la principal diferencia entre estos dos tipos de extractivismo, se encuentra en la participación del Estado, quien entraría a actuar, fundamentalmente, como un agente de redistribución. No obstante, el debate sobre las diferencias que pueden existir entre estos dos tipos de extractivismo está abierto, razón por la cual, este es uno de los aportes de esta investigación.

Para entender las principales diferencias entre el extractivismo clásico y el neoextractivismo, en primer lugar, se analizan los aportes de la literatura sobre este tema, y posteriormente, se contrastan los cambios introducidos en el marco regulatorio del sector petrolero ${ }^{4}$ en dos de los países señalados anteriormente: Colombia y Ecuador. Estas dos partes conforman la estructura de este documento.

\section{REVISIÓN DE LITERATURA}

En este apartado se presentan los elementos conceptuales que caracterizan los dos tipos de extractivismo previamente planteados. Se trata de seguir una estructura que permita identificar las principales diferencias. También se aportan algunos matices dado que no hay un total acuerdo en los rasgos específicos, ni mucho menos en los alcances que tendría cada uno de estos modelos de explotación de RRNN. Las conclusiones obtenidas en la revisión de literatura se convierten en el punto de partida para el análisis comparado del modelo de explotación petrolera ${ }^{5}$ implementado en cada uno de los países seleccionados.

\subsection{Extractivismo}

El uso del término "extractivismo" se ha venido difundiendo en la literatura latinoamericana sin existir precisión acerca del mismo, y por tanto, se ha relacionado con diversas actividades asociadas a la explotación de RRNN. De ahí la necesidad

4. Se toma como referencia el sector petrolero, en primer lugar, por la importancia para la economía de los dos países, y en segundo lugar, por la funcionalidad para el contraste entre los dos tipos de extractivismo.

5. Se entiende como modelo de explotación petrolera, el conjunto de rasgos que caracterizan esta actividad productiva, cuyas bases vienen dadas por el marco regulatorio y la política sectorial de cada país. Esta investigación aborda los aspectos relacionados, únicamente, con el marco regulatorio. 
de acotar lo que implica realmente este concepto, que se asocia fundamentalmente a la minería y al petróleo (Gudynas, 2013; Seoane, 2012).

Partiendo de los planteamientos de Gudynas (2009a, 2011a, 2011b, 2012, 2013), Acosta (2011b), Seoane (2012) y Svampa (2011a y 2011b), los rasgos fundamentales que caracterizan al extractivismo son: a) explotación intensiva o a gran escala de RRNN, tanto renovables como no renovables; b) grado de procesamiento nulo o mínimo; c) los bienes que se extraen se destinan fundamentalmente hacia la exportación.

La orientación exportadora juega un rol fundamental, en tanto que es el canal que da lugar a la subordinación y dependencia. La actividad extractiva representa la primera fase de las cadenas de producción internacionales, con lo cual, la industrialización de las materias primas que son exportadas se realiza fuera de la economía doméstica (Gudynas, 2013).

La forma como se articula el extractivismo a la estrategia de desarrollo, presenta diferencias entre algunos de los países de AL. Las políticas aplicadas a partir de la década de los 2000, reflejan distintos modelos de explotación de RRNN. De ahí que Gudynas (2012 y 2013) señala la existencia de dos tipos de extractivismo: el convencional o clásico, y el neoextractivismo. Siguiendo esta clasificación, se describen las características de cada caso. Cabe advertir que autores como Dávalos (2013a y 2013b) no hacen este tipo de distinción, consideran que solo es posible hablar de extractivismo.

\subsubsection{Extractivismo convencional o clásico}

Es el tipo de extractivismo desarrollado en la mayoría de países de AL durante las últimas décadas, y que en la actualidad se observa en países como Colombia y Perú (Gudynas, 2011c y 2012). El objetivo fundamental dentro del extractivismo clásico es alcanzar altas tasas de crecimiento económico a través del estímulo de las exportaciones. En este marco, la IED en sectores como la minería y los hidrocarburos resulta fundamental (Gudynas, 2010, 2011c, 2012; Centro Latino Americano de Ecología Social, 2008). Para incentivar dichas actividades se ofrecen unas condiciones ventajosas, con las cuales, se logra atraer capitales que permiten incrementar los niveles de producción en periodos de tiempo relativamente cortos. Las medidas adoptadas para alcanzar estos objetivos son: disminución de la carga impositiva, otorgamiento de facilidades para la repatriación de utilidades, reducción de las exigencias medioambientales y laborales etc. (Gudynas, 2010; Acosta, 2010).

Hay una gran confianza en que los mecanismos de mercado son capaces de difundir los efectos positivos desde las actividades extractivas hacia el conjunto de la economía. Se considera que la inversión en estos sectores podría generar encadenamientos con otras actividades, posibilitando así, el efecto derrame o chorreo (Banco Mundial, 2010; Gudynas, 2012 y 2013). Además, de forma directa o indirecta, existe consenso en que las condiciones del mercado mundial prevalecerán, fundamentalmente por el impulso de la demanda de los países emergentes como China e India, que unido a la gran disponibilidad de reservas y su distribución geográfica asimétrica, permiten mantener unos términos de intercambio favorables. 
En el extractivismo convencional el Estado asume un papel pasivo, que se restringe a garantizar las condiciones básicas, tales como, flexibilización laboral, ambiental, tributaria, movimientos de capital etc. (Gudynas, 2009a y 2009b). Las políticas se adecuan a las necesidades del capital privado (transnacionales) y se establece como objetivo propio el crecimiento de la economía. La participación del fisco en la renta generada por las actividades extractivas es baja respecto al valor de la producción generada. Sin embargo, en el conjunto de la estructura de ingresos públicos, suele ser significativa, por lo cual, se busca su incremento por extensión, es decir, aumentando la producción. Bajo esta lógica, disminuyendo la presión fiscal se incrementarían los ingresos (Gudynas, 2013).

La afluencia de grandes inversiones genera un incremento temporal de los ingresos públicos. Este fenómeno puede dar lugar al surgimiento y consolidación de prácticas rentistas, donde los ingresos estatales, poco a poco, pasan a depender de los recaudos derivados de las actividades extractivas ${ }^{6}$. A su vez, el gasto público se convierte en un simple instrumento político, que da lugar a asignaciones discrecionales que terminan favoreciendo, únicamente, a ciertos grupos de la población (Schuldt y Acosta, 2006; Gudynas, 2009a).

Bajo el extractivismo clásico, el papel protagonista lo asumen las empresas transnacionales (Seoane, 2012). La relación que se establece entre este tipo de empresas y los gobiernos es asimétrica, en el sentido de que las primeras imponen sus condiciones, aprovechando las necesidades que tienen estos países. Dicha condición queda reflejada en sus debilidades estructurales, tales como: escasez de capital, temores por desajustes en la balanza de pagos, problemas fiscales, desempleo etc. (Acosta, 2011b).

Debido al carácter que adopta el extractivismo, ha sido objeto de muchas críticas, entendiendo que prevalecen las barreras que coartan los beneficios que deberían generarse en el conjunto de la economía. De la misma manera, queda sin aclarar qué posibilidad hay de alcanzar el desarrollo económico sin una estrategia deliberada en este sentido. De ahí la preocupación sobre los verdaderos alcances de este modelo de explotación de RRNN. Los principales aportes que se citan en esta línea son: las consideraciones contempladas bajo la denominada "enfermedad holandesa"; los planteamientos de la "maldición de los recursos naturales"; y por último, las contribuciones realizadas por los institucionalistas ${ }^{7}$.

A pesar de que los enfoques señalados anteriormente tienen un gran respaldo tanto teórico como empírico, desde una posición ortodoxa se ha buscado rebatirlos, en una clara defensa de la estrategia extractivista. El principal argumento está en que la dotación de RRNN podría convertirse en una plataforma de lanzamiento

6. Aunque rentismo y extractivismo, no significan lo mismo. El rentismo es un concepto más acotado, que implica que el Estado adopta un tipo de comportamiento cuyo objetivo final es apropiarse de la mayor parte de la renta que generan los recursos naturales (hidrocarburos y minería). Bien podría darse el extractivismo sin haber rentismo. Para ampliar la información sobre el rentismo, ver, Mañe y De la Cámara (2005).

7. Dados los objetivos de esta investigación, no se abordan los planteamientos y diferencias en los aportes realizados a partir de estos enfoques. Para ampliar información, ver, Sachs y Warner (1995), Melhum et al. (2006), Corden y Neary (1982) y Krugman (1987). 
que impulsa a otras actividades productivas. Respaldando esta idea, se citan como ejemplos a aquellos países que han tenido experiencias exitosas en este sentido. Los casos que se relacionan son: Inglaterra, Estados Unidos, Canadá, Australia y Noruega (Banco Mundial, 2010; Melhum et al., 2006).

\subsubsection{Neoextractivismo}

A partir de las limitaciones planteadas al extractivismo clásico o convencional, pero con la idea de no renunciar a la explotación de los RRNN, y más en un periodo de bonanza de precios (década de los 2000), surgieron una sería de propuestas que conllevan a confrontar dichas barreras. Se empieza cuestionando los planteamientos ortodoxos, donde el mercado, por sí solo, permite una asignación eficiente de los recursos. La idea central es que los fallos que existen son muy grandes; de lo que se trataría es de corregirlos, o por lo menos, minimizarlos (Acosta, 2009a).

Bajo el argumento de los fallos de mercado, la intervención del Estado resulta fundamental, particularmente en aquellos sectores que se consideran estratégicos, tales como, la minería y los hidrocarburos. Por la importancia en el conjunto de la economía de algunos países, la forma y características que adquiere el modelo de explotación de RRNN y en concreto, en el sector petrolero, redundará en su estilo de desarrollo.

En el marco expuesto anteriormente, se empieza a configurar una nueva variante de extractivismo. Gudynas (2013) acuñó el término "neoextractivismo" bajo una coyuntura particular desarrollada en América Latina a mediados de la década de los 2000, caracterizada por: auge fuerte y prolongado en los precios de las materias primas, particularmente mineras e hidrocarburíferas; tasas de crecimiento económico relativamente altas; y por último, surgimiento de movimientos políticos de carácter progresista en países como Venezuela, Bolivia, Ecuador y Uruguay. De ahí que también se haya denominado como neoextractivismo progresista del siglo XXI (Gudynas, 2012).

Para Gudynas (2009a, 2011a, 2011c), el neoextractivismo reproduce los cimientos básicos bajo los cuales funciona el extractivismo clásico o colonial, la diferencia fundamental estaría en la presencia del Estado. Como se señaló anteriormente, no hay esa fe ciega en que los mecanismos de mercado vayan a ser capaces de generar, por si solos, efectos positivos hacia el conjunto de la economía, y a la vez, contribuir al desarrollo económico de los países. Así, la acción del Estado estaría plenamente justificada. Los principales mecanismos de intervención son: participación directa en la producción, donde juegan un rol fundamental las empresas públicas; mayor presión fiscal, que permite que se capte una mayor parte de la renta generada por la explotación de los RRNN; y por último, el desarrollo de instrumentos de regulación, lo cual reduce el impacto negativo que se puede causar en materia ambiental, social etc.

Bajo el neoextractivismo, el Estado es mucho más fuerte y activo, contrario a la función adoptada en el extractivismo clásico. A partir de las nuevas funciones, el Estado es capaz de garantizar el efecto derrame o goteo. Pero no solamente se queda conforme con dicha función, se establecen mecanismos para resarcir a quienes no se benefician, cosa que el mercado, por sí solo, no haría (Composto y Navarro, 2012). 
Para Gudynas "este es una especie de capitalismo benévolo que apunta a lidiar con la pobreza y la desigualdad con rectificaciones y compensaciones” (2011d: 38).

La intervención del Estado genera dos efectos importantes: primero, un incremento de las rentas públicas (en forma de regalías, impuestos etc.); y segundo, el control en la forma como se hace la explotación de los recursos naturales (Hollender, 2012; Gudynas, 2012; Acosta, 2011b; Composto y Navarro, 2012). Con los recursos captados se fortalece la capacidad de paliar los efectos negativos que dichas actividades generan, surgiendo así una especie de "Estado compensador", lo cual profundiza su papel como agente de redistribución (Svampa, 2013; Gudynas, 2012).

El uso que se le pueda dar a la renta extractiva se asume como un paso fundamental de la transición hacia una economía postextractivista. La dependencia fiscal sobre los recursos generados en la explotación de los recursos naturales, no sería un problema per se, por tanto, la profundización de las actividades mineras y de hidrocarburos se vería como algo necesario (Gudynas, 2011a y 2011c; Dávalos, 2013a). Desde otro punto de vista "la renta extractiva se convierte en una estrategia de articulación social y política que permite movilizar a la sociedad y generar un consenso sobre el extractivismo como necesidad ineludible para el desarrollo y la equidad” (Semplandes, 2009; citado por Dávalos, 2013a: 4). En el mismo sentido, Svampa (2013) se refiere a un "consenso de las commodities", donde el neoextractivismo sería un sinónimo de crecimiento, estabilidad, redistribución y lucha contra la pobreza.

No obstante, aunque se resalta la importancia del Estado en el proceso de distribución de la renta, su función va más allá. La idea fundamental es configurar una estrategia de desarrollo a partir de la explotación de los RRNN. La intervención estatal estará orientada a realizar un mejor proceso de asignación de recursos entre las distintas actividades económicas, que se complementa con los mecanismos de distribución del ingreso (Pérez, 2010). Hay una gran confianza en que al adoptar unas políticas adecuadas, se puede romper con el círculo vicioso al cual se han enfrentado muchos de los países ricos en RRNN.

Los ingresos provenientes de la explotación de los RRNN se convierten en una fuente fundamental de financiamiento, y con el tiempo, pueden contribuir a reducir el peso de las actividades extractivas. En esta línea, Intermón Oxfam (2011) insiste en que los países exitosos, que han pasado por auges en bienes primarios, lo han sido, debido al buen manejo que le han dado a los recursos fiscales.

Los planteamientos realizados anteriormente mostraron que el neoextractivismo, efectivamente, plantea algunos cambios importantes respecto al extractivismo clásico. Sin embargo, autores como Gudynas (2013 y 2011c), Acosta (2011b) y Dávalos (2013b), aun aceptando la importancia de las políticas implementadas por varios de los gobiernos progresistas en AL durante la década de los 2000, consideran que a partir del neoextractivismo no existe la posibilidad de una transición hacia un modelo de acumulación diferente. De ahí que asuman que por las propias limitaciones estructurales, entre ellas, el patrón de inserción externa, aunadas a una estrategia del Estado centrada en la redistribución de la renta, dicho escenario, fácilmente quedará truncado. 
Para Gudynas (2011e), bajo el neoextractivismo, lo que se pone en cuestión del llamado extractivismo clásico es el control privado que se ejerce sobre los recursos naturales y los beneficios que se obtienen, no la explotación como tal. De hecho, la presencia de las transnacionales continúa, pero bajo una modalidad diferente (Gudynas, 2012). El Estado pasa a ser un actor con mayor relevancia, aun así, no es suficiente para emprender una estrategia viable de desarrollo. El problema está en que no se cuestiona el funcionamiento ni los límites del extractivismo, las relaciones económicas internacionales, la forma como se fijan los precios de las commodities, mucho menos, la posición de la economía frente al resto del mundo (Monge, 2012; Carrasco y Del Hoyo Arce, 2012).

Para Gudynas (2013), el carácter dependiente en ningún momento se modifica, la existencia de cadenas globales de comercialización de materias primas hace que la demanda y los precios se determinen en el exterior. De ahí que la intervención del Estado, nuevamente, quede limitada en términos de potenciar una nueva estrategia productiva, entretanto, continua siendo funcional al mercado global de materias primas.

Así, mientras para el extractivismo clásico la justificación para profundizar este modelo de explotación de RRNN es el crecimiento económico, para el neoextractivismo serían las políticas públicas; que es posible financiarlas gracias a las rentas generadas por la explotación de las actividades extractivas (Composto y Navarro, 2012). De ahí que Acosta (2012) señala que lo que en realidad se está haciendo es reinventar el extractivismo, acompañándolo con algo de keynesianismo.

\section{ANÁLISIS DE LOS MODELOS DE EXPLOTACIÓN PETROLERA DE COLOMBIA Y ECUADOR: APROXIMACIÓN DESDE EL MARCO REGULATORIO}

Teniendo en cuenta las conclusiones de la revisión de literatura, la comparación de los dos tipos de extractivismo se realiza a partir del análisis de las principales modificaciones introducidas en el marco regulatorio del sector petrolero en los dos países seleccionados. Por las consideraciones anotadas en la parte inicial de este documento, el periodo de referencia para el análisis es la década de los 2000.

La premisa de partida es que, si el elemento diferencial entre el extractivismo clásico y el neoextractivismo gira en torno a la presencia del Estado, la expresión concreta del rol que este debe cumplir dentro de la economía se refleja en el marco regulatorio. Para los objetivos planteados dentro esta investigación resulta fundamental su comprensión dado que es el primer acercamiento al modelo de explotación petrolera implementado en cada uno de los países.

La idea fundamental que se plantea es que la organización y cambios realizados en el sector petrolero reflejan dos modelos de explotación distintos, que sin ser totalmente antagonistas, claramente muestran diferencias. Como rasgo general, a lo largo de la década de los 2000, tanto en Colombia como en Ecuador se presentan modificaciones importantes en el funcionamiento del sector petrolero, aunque los objetivos perseguidos en cada caso reflejan situaciones completamente distintas.

En Ecuador, los cambios en materia de regulación obedecen a la necesidad de recobrar la importancia estatal, empezando por una mayor participación en 
la renta petrolera, que también está concatenada con la modificación en la forma como se establecen las relaciones con las empresas privadas. Lo que se pretendió desde 2006 es recuperar la participación del Estado, dada la posición que habían adoptado las transnacionales, y los bajos márgenes que, a su juicio, se pagaban por concepto de impuestos (Ramírez, 2011). A través de estos instrumentos, el Estado intenta ejercer un mayor control sobre el sector petrolero.

En Colombia, por el contrario, se apuesta por un mayor grado de apertura del sector. Los cambios realizados en el marco regulatorio buscan alcanzar dos objetivos: incrementar la producción y, aumentar el volumen de reservas petroleras. Dentro de las estrategias planteadas, las medidas para atraer inversión privada resultan fundamentales (Unidad de Planeación Minero Energética [UPME], 2013). Asimismo, se toman decisiones tendientes a mejorar la gestión de Ecopetrol. En este marco, las relaciones Estado-empresas se refuerzan, de ahí que la estructura institucional y contractual sea completamente favorable a la entrada de capital privado.

Una vez expuestos los objetivos por los cuales se realizan las modificaciones del marco regulatorio en cada uno de estos países, se pasa a revisar los elementos concretos de este proceso. Inicialmente se presenta una descripción de las cuestiones institucionales y contractuales, y luego, se analizan las disposiciones en materia de captación y uso de la renta petrolera.

\subsection{Cambios institucionales y contractuales}

Las principales reformas institucionales dentro del sector petrolero colombiano se realizaron en la primera parte de la década de los 2000. Los cambios más importantes son: en primer lugar, se separan las funciones estatales; y en segundo lugar, se define el carácter que adquiere Ecopetrol. Bajo estas directrices, en el año 2003, se crearon, la Agencia Nacional de Hidrocarburos (ANH) y la Sociedad Promotora de Energía de Colombia S.A. ${ }^{8}$. A partir de ese momento, Ecopetrol pasó a ser una empresa pública por acciones, concentrándose en labores netamente operativas y dejando en manos de la ANH la gestión administrativa y de control.

En el caso de Ecuador, se presentan algunas semejanzas, no obstante, hay diferencias en el marco institucional. La separación de actividades de gestión y producción fue realizada en el año 2010, cuando se crean, la Agencia de Regulación y Control Hidrocarburífero (ARCH) y, la Secretaría de Hidrocarburos (SH) ${ }^{9}$. Sin embargo, EP Petroecuador mantiene su estructura, la cual está muy ligada a las directrices provenientes de la rama ejecutiva, quien es responsable de la política de hidrocarburos, a la vez que tiene injerencia en las decisiones de la empresa.

En Colombia, a la vez que se presentan cambios institucionales, paralelamente se modifican los esquemas de contratación. El cambio más importante se introduce con la reforma de 2003, donde se pasa de los contratos de asociación a esquemas

8. Decreto No. 1760 del 26 de junio de 2003.

9. Se separan la gestión de contratos que queda en manos de la SH y las actividades de fiscalización que asume la ARCH. Artículo 11 Ley de Hidrocarburos. Registro Oficial No.244 del 27 de Julio del 2010. 
de concesión (Campodónico, 2007; UPME, 2013). Desde 1974 se había tenido un mecanismo a través del cual, Ecopetrol, ante el descubrimiento de un yacimiento, se convertía en socio de los nuevos contratos, con una participación del 50\%, que en el año 2000 se redujo al 30\%. A partir de 2004, con el nuevo esquema, las empresas privadas que desarrollen actividades de exploración ya no están obligadas a asociarse con Ecopetrol en ninguna de las fases del proceso de explotación petrolera ${ }^{\mathbf{1 0}}$.

Además, no hay ninguna preferencia para las empresas públicas, es decir, Ecopetrol "puja" por las ofertas existentes como un actor más. De otro lado, las empresas "concesionarias", luego de pagar las regalías, pueden disponer libremente de la producción de crudo (o, si fuera el caso, de derivados), siendo un estímulo más para la inversión privada. Tampoco hay grandes exigencias asociadas a los contratos, ni en términos de tecnología, contratación nacional, reinversiones, etc. ${ }^{\mathbf{1 1}}$. Aquí estaría plasmada una de las características del extractivismo clásico, donde el Estado busca atraer inversión privada a través de estímulos de diverso tipo.

La situación de Ecuador dista mucho de la planteada para el caso colombiano. Las reformas se realizaron, como se dijo anteriormente, tratando de maximizar el "beneficio" a favor del Estado, fundamentalmente, a través de la participación en la renta petrolera. Bajo este argumento, se modificó el esquema de contratación, lo cual implicó el paso desde los contratos de participación a los de prestación de servicios.

Por el impacto económico y por la relación que se establece entre las empresas y el Estado, el cambio que se genera es muy drástico. No solamente se trata de incrementar el recaudo estatal, como efectivamente sucedió, también se busca fortalecer una figura contractual donde el Estado deja muy clara la propiedad sobre el recurso energético. Esto no quiere decir que las empresas privadas fueran absorbidas por el Estado, de hecho no se puede hablar de nacionalización. Lo que se hace es cambiar las reglas del juego.

Por otra parte, a diferencia de Colombia, la favorabilidad hacia empresas públicas es taxativa; no obstante, pueden haber licitaciones donde participen otro tipo de empresas. Además, sí hay algunas exigencias colaterales para las empresas que participan en cualquier etapa de la cadena de producción, tales como: contratación de mano de obra local (cualificada y no cualificada), reinversión de utilidades y cumplimiento de programas de inversión. Por último, las empresas que participen en procesos de producción, están obligadas a entregar el crudo a EP Petroecuador quien es la empresa encargada, directa o indirectamente, de realizar la comercialización.

De esta manera, las medidas implementadas en Ecuador son concordantes con los planteamientos realizados en el marco del neoextractivismo, en la medida en que el Estado se va convirtiendo en un actor de mayor relevancia dentro de la actividad petrolera.

10. Todos los contratos firmados hasta 2003 bajo la modalidad de asociación se mantienen hasta que se agoten las reservas.

11. Aunque se paga una tasa por transferencia de tecnología, cuyo recaudo corresponde a la ANH. 


\section{Cuadro 1 \\ SÍNTESIS DE LAS MODIFICACIONES EN LOS ESQUEMAS CONTRACTUALES E INSTITUCIONALES}

\begin{tabular}{|c|c|c|}
\hline Criterio & Colombia & Ecuador \\
\hline $\begin{array}{l}\text { Años en que se realizan las } \\
\text { principales reformas en el } \\
\text { sector petrolero. }\end{array}$ & $2002-2003$ & $2006-2010$ \\
\hline $\begin{array}{l}\text { Tipo de contrato predomi- } \\
\text { nante (nuevos contratos). }\end{array}$ & Concesión. & Prestación de servicios. \\
\hline $\begin{array}{l}\text { Forma de adjudicación de } \\
\text { bloques petroleros. }\end{array}$ & Rondas de licitación pública. & $\begin{array}{l}\text { Directa. Como mecanismo } \\
\text { adicional existe la posibili- } \\
\text { dad de realizar este proceso } \\
\text { a través de rondas de licita- } \\
\text { ción pública. }\end{array}$ \\
\hline $\begin{array}{l}\text { Preferencia por empresas } \\
\text { públicas en los procesos de } \\
\text { contratación. }\end{array}$ & No. & Si. \\
\hline $\begin{array}{l}\text { Naturaleza jurídica de la } \\
\text { principal empresa pública. }\end{array}$ & Empresa de economía mixta. & Empresa estatal. \\
\hline $\begin{array}{l}\text { Exigencias colaterales a las } \\
\text { empresas que participan } \\
\text { en labores de exploración y } \\
\text { explotación. }\end{array}$ & $\begin{array}{l}\text { Cumplimiento programas de } \\
\text { inversión. }\end{array}$ & $\begin{array}{l}\text { Contratación de mano de } \\
\text { obra local (cualificada y no } \\
\text { cualificada), reinversión de } \\
\text { utilidades, cumplimiento de } \\
\text { programas de inversión, etc. }\end{array}$ \\
\hline $\begin{array}{l}\text { Propiedad del crudo pro- } \\
\text { ducido. }\end{array}$ & $\begin{array}{l}\text { Empresa que hace la explo- } \\
\text { tación. }\end{array}$ & Estado. \\
\hline $\begin{array}{l}\text { Entidad encargada de reali- } \\
\text { zar labores de fiscalización. }\end{array}$ & $\begin{array}{l}\text { Agencia Nacional de Hidro- } \\
\text { carburos }(\mathrm{ANH}) \text {. }\end{array}$ & $\begin{array}{l}\text { Agencia de Regulación y } \\
\text { Control Hidrocarburífero } \\
(\mathrm{ARCH}) \text {. }\end{array}$ \\
\hline
\end{tabular}

Fuente: elaboración propia.

Si se observa la cantidad de contratos firmados después de las modificaciones en el marco regulatorio, se puede tener una idea del impacto que han tenido los nuevos esquemas. Sin embargo, las conclusiones pueden verse distorsionadas por un escenario marcado por el incremento en los precios del petróleo. Bajo estas consideraciones, se pasa a tipificar los aspectos contractuales de los países seleccionados.

En Colombia, bajo el nuevo esquema de contratación, que partir de 2004 es la “concesión”, existen tres modalidades: exploración y producción (E\&P), evaluación 
técnica (TEA) y, especial. Entre 2004 y 2012 el número de contratos firmados bajo la modalidad de E\&P fue de 349. En el mismo periodo se firmaron 97 contratos de evaluación técnica. Entre tanto, bajo la modalidad de asociación, que corresponde al sistema vigente hasta 2003, se firmaron un total de 312 contratos (periodo 2004-2009) ${ }^{\mathbf{1 2}}$.

En el caso ecuatoriano, el giro que se hace en materia de contratación tiene un sentido completamente distinto. Bajo la modalidad de prestación de servicios, que representa el esquema contractual que pasa a predominar, lo que hace el Estado es asignar un área o bloque ya sea para la exploración y/o explotación, y luego, paga a las empresas una tarifa por barril producido. Los contratos que estaban vigentes a 2007, fueron renegociados, y pasaron a la modalidad de prestación de servicios, proceso que culminó en el año 2010. En el Cuadro 1 se presenta una síntesis de los principales cambios en esta materia.

\subsection{Participación y uso de la renta petrolera}

\subsubsection{Participación en la renta petrolera}

En Ecuador, dentro de ese gran vuelco en el modelo de explotación petrolera, se cambiaron las condiciones en cuanto a la participación del Estado en la renta. Con la reformas a la Ley de Hidrocarburos realizadas en el año 2006, y posteriormente en 2010, queda claro dicho objetivo. En el año 2006, estando vigentes los contratos de participación, se obligó a que las empresas reconocieran a favor del Estado, el 50\% de los ingresos extraordinarios ${ }^{13}$. Luego, en 2007, con Rafael Correa en la presidencia, dicho porcentaje se elevó al 99\% ${ }^{14}$. A partir de 2008, se entró a renegociar los contratos para cambiarlos a la modalidad de prestación de servicios, con lo cual, el Estado, a través de EP Petroecuador, capta la diferencia entre la tarifa pactada y el precio de venta del crudo. Además, se establece una participación del $25 \%$ que corresponde a lo que se denominó como el "margen de soberanía", que se calcula sobre los ingresos brutos de la actividad.

En el caso de Colombia la situación es completamente opuesta. La reforma contractual implicó una disminución en la participación del Estado. Con la legislación anterior, las empresas tenían que pagar una regalía equivalente al $20 \%$, independientemente del tamaño del yacimiento. A partir de la Ley 756 de 2002, se establece una tarifa escalonada que va del $8 \%$ al $25 \%$, y depende del nivel de producción ${ }^{15}$. No obstante, a pesar del crecimiento en los precios del crudo en los años posteriores, no hubo ninguna modificación de facto que buscara aumentar la participación estatal.

12. Información obtenida de la ANH y el Sistema de Información de Petróleo y Gas (SIPG).

13. Se entiende como la diferencia entre el precio promedio mensual efectivo de venta FOB del petróleo crudo ecuatoriano y el precio promedio mensual de venta vigente a la fecha de suscripción del contrato.

14. Decreto ejecutivo No. 662 de 2007. De todas maneras, en 2008, se modificó al 70\% (Ramírez, 2011)

15. Solamente aquellas explotaciones que producen más de 600 mil barriles diarios pagarían la tarifa del $25 \%$, algo que no sucede en Colombia. 


\subsubsection{Uso de la renta petrolera}

Como se anotó en la revisión de la literatura, la forma como el Estado hace uso de las rentas derivadas de los recursos naturales es un elemento fundamental para reducir el peso de las actividades extractivas. El papel de las instituciones es decisivo dentro de este proceso (Melhum et al., 2006).

En el caso de Ecuador, hay una secuencia clara entre los cambios institucionales, contractuales y fiscales. En este marco de reformas, se tramitó, en 2008, la "Ley orgánica para la recuperación del uso de los recursos petroleros del Estado y la racionalización administrativa de los procesos de endeudamiento". Las implicaciones más importantes son: la concentración de los recursos en el nivel central, la prohibición de utilizar los ingresos petroleros para financiar gasto corriente, y por último, la eliminación de algunos fondos que venían en periodos anteriores. No obstante, a pesar que se eliminaron dichos fondos, no se crea uno nuevo. Ahora bien, aunque son muy claras las medidas en el tema del recaudo, no sucede lo mismo en el caso de los gastos. Así pues, dado que en el presupuesto de inversión no se discrimina por fuente de financiamiento, no se podría conocer con exactitud el destino de los ingresos petroleros.

En el caso colombiano también se hace complejo identificar la forma como se usan los recursos petroleros. El problema es que no existe una asignación específica para los recaudos por concepto de renta (sociedades) o los dividendos que le paga Ecopetrol al Estado. El único caso donde hay unas directrices específicas es en las regalías, que están dirigidas al financiamiento de proyectos regionales ${ }^{\mathbf{1 6}}$. Empero, han existido dificultades en su manejo, debido a su grado de dispersión (por la cantidad de beneficiarios) y la falta de control y seguimiento a los recursos asignados.

La revisión del marco regulatorio permitió un primer acercamiento a los cambios introducidos durante los últimos años en los modelos de explotación petrolera de los dos países. Dichas modificaciones son consistentes con los argumentos señalados dentro de las dos variantes de extractivismo desarrolladas en la revisión de literatura. En el caso Ecuatoriano, es clara la posición del Estado por recuperar el control de la actividad petrolera, que se expresa a través de disposiciones en cuanto al rol que entran a jugar las empresas públicas, la búsqueda de una mayor participación en la renta petrolera y la modificación en las relación con las empresas transnacionales, que se refleja en el cambio en los esquemas de contratación y las exigencias colaterales. No obstante, frente al uso de la renta petrolera, no se pueden sacar grandes conclusiones.

En el caso colombiano, el rasgo más importante viene dado por un mayor grado de apertura del sector, donde hay una política agresiva para atraer IED, lo cual ha implicado, medidas de tipo impositivo, cambios en los esquemas de contratación, una menor participación del Estado a través de Ecopetrol etc. Los aspectos aludidos guardan coherencia con las características descritas en el extractivismo clásico. En el Cuadro 2 se presenta una síntesis los principales cambios en este aspecto.

16. Ver Acto Legislativo No. 05 de 2011. 


\section{Cuadro 2}

\section{SÍNTESIS DE LAS MODIFICACIONES EN LA PARTICIPACIÓN Y USO DE LA RENTA PETROLERA}

\begin{tabular}{|c|c|c|}
\hline Criterio & Colombia & Ecuador \\
\hline Tarifa general de regalías (\%). & Rango que va del $8 \%$ al $25 \%$. & $\begin{array}{l}\text { Rango que va del } 12.5 \% \text { al } \\
18.5 \% \text {. }\end{array}$ \\
\hline $\begin{array}{l}\text { Impuesto sobre la renta (ta- } \\
\text { rifa general). }\end{array}$ & $\begin{array}{l}\text { Impuesto a las sociedades: } \\
25 \% \text {. } \\
\text { Impuesto sobre la renta } \\
\text { para la equidad (CREE): el } \\
9 \% \text { para el período 2013- } \\
2015 \text { y luego el } 8 \% \text {. }\end{array}$ & $23 \%$. \\
\hline $\begin{array}{l}\text { Otros impuestos sobre los } \\
\text { ingresos petroleros. }\end{array}$ & No aplica. & $\begin{array}{l}\text { Al menos el } 50 \% \text { de los } \\
\text { ingresos extraordinarios } \\
\text { (contratos de participación). } \\
\text { Margen de soberanía } 25 \% \text {. }\end{array}$ \\
\hline Participación pública. & Ecopetrol. & Petroecuador. \\
\hline $\begin{array}{l}\text { Tipo de gastos que se finan- } \\
\text { cian con ingresos provenien- } \\
\text { tes de la participación en la } \\
\text { renta petrolera. }\end{array}$ & Inversión - gastos corrientes. & $\begin{array}{l}\text { Inversión - subsidios a los } \\
\text { combustibles. }\end{array}$ \\
\hline $\begin{array}{l}\text { Disposiciones concretas para } \\
\text { el uso de la renta petrolera. }\end{array}$ & $\begin{array}{l}\text { Solamente en el caso de las } \\
\text { regalías y fondos especiales. } \\
\text { Fondos: Ciencia, Tecnología } \\
\text { e Innovación; de Desarrollo } \\
\text { Regional; de Compensación } \\
\text { Regional; y de Ahorro y Es- } \\
\text { tabilización. }\end{array}$ & $\begin{array}{l}\text { Solamente para algunos } \\
\text { fondos especiales. Fondo } \\
\text { Ecodesarrollo. }\end{array}$ \\
\hline
\end{tabular}

Fuente: elaboración propia.

\section{CONCLUSIONES}

Uno de los objetivos propuestos dentro de esta investigación fue, contribuir al análisis sobre las diferencias entre las dos variantes del extractivismo señaladas por Gudynas (2011a, 2011c, 2012). Con este fin, se hizo, en una primera parte, la revisión de los principales aportes frente a este tema, y en segundo lugar, se realizó la comparación de los cambios introducidos en el marco regulatorio del sector petrolero en dos países seleccionados.

Estando de acuerdo en que existen elementos comunes entre el extractivismo clásico y el neoextractivismo, un rasgo diferencial importante entre estos dos modelos de explotación de los RRNN es, sin lugar a dudas, el papel que asume el 
Estado. No obstante, la intervención estatal va más allá de la captación de la renta con objeto de redistribuirla en programas sociales. Aunque no sería ilógico que se hiciera, dadas las necesidades y carencias a las cuales se enfrentan los países latinoamericanos.

El neoextractivismo implica un cambio en el modelo de explotación de los RRNN y, particularmente, del petróleo. Se intenta, por una parte, captar una mayor parte de la renta petrolera, y por otra, darle un uso eficiente a la misma. Una proporción de esa renta se destinaría a fortalecer el modelo productivo (explotación de recursos naturales), y el excedente, al desarrollo de programas sociales. La idea es que, los cambios estructurales no se darán en el corto plazo, y por tanto, para transitar hacia un escenario menos dependiente del extractivismo, puede ser necesario, en una primera etapa, la profundización en la explotación. Este concepto plantearía una diferencia clave entre el extractivismo clásico y el neoextractivismo.

El rol que juega el Estado es fundamental bajo el neoextractivismo. Los mecanismos de intervención son diversos y van desde la influencia que pueda ejercer por la vía del marco regulatorio hasta la actuación directa a través de las empresas públicas. No se trata solamente de participar en la cadena de producción como un agente más; el Estado busca convertirse en un actor de peso en la configuración y funcionamiento del sector. Dicha condición ofrece la posibilidad de establecer un modelo que, sin desconocer las limitaciones a las que se enfrenta, intenta corregir o mitigar aquellos obstáculos con los que han tenido que lidiar, históricamente, los países que se especializan en la explotación de los RRNN, donde las experiencias, en la mayoría de los casos, no han sido exitosas.

Por otra parte, aunque se insistió en la importancia que tiene el uso de la renta petrolera dentro del neoextractivismo, las modificaciones introducidas en el marco regulatorio no permiten realizar una valoración en términos de eficiencia. En el caso ecuatoriano, donde se suponía, debe haber medidas más claras en este sentido, no es posible conocer la manera como son utilizados los recursos petroleros. Al no tener mecanismos que indiquen los gastos realizados con esta fuente de financiamiento, se hace muy difícil conocer su impacto.

En el caso colombiano, su modelo de explotación petrolera refleja algunos rasgos que fueron descritos bajo el denominado extractivismo clásico. Como se pudo ver, se estructuró un marco regulatorio orientado a la atracción de capital privado, fundamentalmente transnacional, que en un contexto de subida de los precios del petróleo, hizo posible una ingente entrada de IED.

Por último, aunque las modificaciones introducidas en el marco regulatorio muestran grandes diferencias en los modelos de explotación petrolera de los dos países, es necesario evaluar la dinámica productiva y fiscal. No se puede desconocer el efecto generado por los cambios en el marco regulatorio, sin embargo, las condiciones previas y los grandes desafíos que implica la configuración de un nuevo modelo de explotación petrolera, sugieren muchas dudas sobre el verdadero potencial de este tipo de estrategias. 


\section{Referencias}

ACOSTA, Alberto. (2009a). La maldición de la abundancia (1a Ed.). Ediciones Abya-Yala. Quito-Ecuador. (2010). Maldiciones que amenazan la democracia, Revista Nueva Sociedad, núm. 229, pp. $42-61$.

(2011b). Extractivismo y neoextractivismo: dos caras de la misma maldición. Más allá del desarrollo (1 $1^{\mathrm{a}}$ Ed.), Grupo permanente de trabajo sobre alternativas al desarrollo, Fundación Rosa Luxemburg, Abya Yala, Quito, pp. 83-118.

(2012). El retorno del Estado. Primeros pasos postneoliberales, más no postcapitalistas, Revista La Tendencia, núm. 13, (abril-mayo), Quito, pp. 62-72.

ALTOMONTE, Hugo; ACQUATELLA, Jean; ARROYO, Andrés y JOURAVLEV, Andrei (2013). Recursos naturales en Unasur: situación y tendencias para una agenda de desarrollo regional, CEPAL, Santiago de Chile.

BANCO MUNDIAL (2010). Los recursos naturales en América Latina y el Caribe, ¿Más allá de las bonanzas y crisis? (1 ${ }^{\text {a }}$ Ed.), Colombia.

CAMPODÓNICO, Humberto (2007). La gestión de la industria de hidrocarburos con predominio de empresas del Estado. CEPAL/GTZ, serie Recursos Naturales e Infraestructura, núm. 121, Santiago de Chile.

CARRASCO, Inés y DEL HOYO ARCE, Jokin (2012). Neoextractivismo, Observatorio de las Multinacionales en América Latina. Disponible en: http://omal.info/IMG/article_PDF /Neoextractivismo_a4847.pdf

CENTRO LATINO AMERICANO DE ECOLOGÍA SOCIAL (2008). Heterodoxos. Tensiones y posibilidades de las políticas sociales en los gobiernos progresistas de América del Sur, Gráficos del Sur. Montevideo.

COMPOSTO, Claudia (2012). Acumulación por despojo y neoextractivismo en América Latina. Una reflexión crítica acerca del estado y los movimientos socio-ambientales en el nuevo siglo. En: Astrolabio, Universidad Nacional de Quilmes, núm. 12, pp. 323-352.

COMPOSTO, Claudia. (2012) y NAVARRO, Mina Lorena. (2012). Estados, transnacionales extractivas y comunidades movilizadas: dominación y resistencias en torno de la minería a gran escala en América Latina. En Revista Theomai, núm. 25 (primer semestre), Buenos Aires.

CORDEN, W. y NEARY, J. (1982). Booming sector and de-industrialitation in a small open economy. The Economic Journal, [e-journal] 92 (368), diciembre de 1982, pp. 825-848.

DÁVALOS, Pablo (2013a). Las falacias del discurso extractivista, América Latina en movimiento. Disponible en: http://alainet.org/active/64266\&lang=es

(2013b). Extractivismo y teoría de las instituciones [En línea] Disponible en: http://www. rebelion.org/noticia.php?id=166338

EMPRESA COLOMBIANA DE PETRÓLEOS [Ecopetrol] (2006). La capitalización de Ecopetrol [En línea] Disponible en: http://www.icpcolombia.org/archivos/ documentos/ capitalizacionminm inas.pdf

GUDYNAS, Eduardo (2009a). Diez tesis urgentes sobre el nuevo extractivismo. Contextos y demandas bajo el progresismo sudamericano actual. En: Extractivismo, política y sociedad, CAAP y CLAES, Quito, pp. 187-225. 
(2009b). Estado y mercado en América Latina: una pareja despareja. En: Revista Nueva Sociedad, núm. 221, pp. 54-65.

(2010). Si eres tan progresista ¿Por qué destruyes la naturaleza?, Neoextractivismo, izquierda y alternativas. En: Revista Ecuador Debate, núm. 79, Quito, pp. 61-81.

(2011a). Más allá del nuevo extractivismo: transiciones sostenibles y alternativas al desarrollo. En: El desarrollo en cuestión, reflexiones desde América Latina, Fernanda Wanderley (Coord.), Oxfam y CIDES UMSA, Bolivia, pp. $379-410$.

(2011b). La maldición de los recursos naturales y las opciones de desarrollo, Semanario Voces. [En línea] Disponible en: http://www.slideshare.net/ ecuadordemocratico/la-maldicin-de-los-recursosnaturales-y-las-opciones-de-desarro llo-por-eduardo-gudynas (Consultado 5 de diciembre de 2013).

(2011c). Alcances y contenidos de las transiciones al Post-Extractivismo. En:EcuadorDebate, núm. 82, Quito, pp. 61-80.

(2011d). Debates sobre el desarrollo y sus alternativas en América Latina: una breve guía heterodoxa. En: Grupo Permanente de Trabajo sobre Alternativas al Desarrollo (eds.): Más allá del desarrollo. Quito: Abya Yala/Fundación Rosa Luxemburg, pp. 21-55.

(2011e). El nuevo extractivismo progresista en América del Sur. Tesis sobre un viejo problema bajo nuevas expresiones. En: Colonialismo del siglo XXI, Negocios extractivos y defensa del territorio en América Latina, Barcelona, pp. 75-92.

(2012). Estado compensador y nuevos extractivismos. Las ambivalencias del progresismo sudamericano. En: Revista Nueva Sociedad, núm. 237, pp. 128-146.

(2013). Extracciones, extractivismos y extrahecciones. Un marco conceptual sobre la apropiación de los recursos naturales. Observatorio del Desarrollo No. 18. Centro Latinoamericano de Ecología Social.

HOLLENDER, Rebecca (2012). Política ambiental de los países progresistas en Latinoamérica. Buen Vivir vs Neo-Extractivismo. Observatorio de multinacionales en América Latina. [En línea] Disponible en: http:// www.biodiversidadla.org/Principal/Secciones/Documentos/Politica_ambiental_de_los_paises_progresistas_en_Latinoamerica_Buen_Vivir_vs._neo-extractivismo

INTERMÓN OXFAM (2009). Contra la "maldición" de los recursos naturales. Informe No. 134.

KRUGMAN, Paul (1987). The narrow moving band, the Dutch disease, and the competitive consequences of Mrs. Thatcher: notes on trade in the presence of dynamic scale economies, Journal of Development Economics, vol. 37, pp. 41-55.

MAÑE, Aurelia y DE LA CÁMARA, Carmen. (2005). Is Russia Drifting towards an Oil Rentier Economy, Eastern European Economics, vol. 43, núm. 5, September-October, pp. 46-73.

MEHLUM, H.; MOENER, K. y TORVIK, R. (2006). Institucions and the Resource Curse, The Economic Journal. 16 (January), pp. 1-20.

MONGE, Carlos. (2012). Extractivismo y postextractivismo en el Perú. Análisis seminario internacional - Desarrollo territorial y extractivismo: luchas y alternativas en la región andina, Centro de Estudios Regionales Andinos Bartolomé de las Casas, Perú, pp. 207-212.

PÉREZ, Carlota. (2010). Dinamismo tecnológico e inclusión social en América Latina: una estrategia de desarrollo productivo basado en los recursos naturales, Revista de la Cepal, núm. 100, Abril, pp. 123-145. 
RAMÍREZ, Juan M. (2011) (Coord.). La explotación de los hidrocarburos y el fomento del desarrollo en América Latina: los casos de Bolivia, Brasil y Ecuador, Grupo de Estudios de Economía Política: Capitalismo y Desarrollo Desigual, Universidad Complutense de Madrid.

SACHS, J. D. y WARNER, A. M. (1995). Natural resource abundance and economic growth, NBER, Working Paper No. 5398.

SCHULDT, Jürgen y ACOSTA, Alberto. (2006). Petróleo, rentismo y subdesarrollo: ¿una maldición sin solución?. En: Revista Nueva Sociedad, núm. 204, Quito, pp. 71-89.

SEOANE, José (2012). Neoliberalismo y ofensiva extractivista. Actualidad de la acumulación por despojo, desafíos de nuestra América. En: Revista Theomai, núm. 26. Segundo semestre de 2012.

SVAMPA, Maristella (2011a). Minería y neoextractivismo latinoamericano. [En línea] Disponible en: http:// huerquenweb.files.wordpress.com/2011/08/minerc3ada_y_neoextractivismo_latinoamericano-svampa. pdf

(2011b). Extractivismo neodesarrollista y movimientos sociales: ¿Un giro ecoterritorial hacia nuevas alternativas?. En: Grupo Permanente de Trabajo sobre Alternativas al Desarrollo (eds.): Más allá del desarrollo. Quito: Abya Yala/Fundación Rosa Luxemburg, 185-218.

SVAMPA, Maristella. (2013). El consenso de las commodities y lenguajes de valoración en AL. En: Revista Nueva Sociedad, núm. 244, (marzo - abril), pp. 30-46.

UNIDAD DE PLANEACIÓN MINERO ENERGÉTICA [UPME] (2013). Cadena del Petróleo 2013. Bogotá. 